\title{
Mortality after road traffic crashes in a system with limited trauma data capability
}

Hassan Saidi ${ }^{1 *}$, Ben Kasyoka Mutiso ${ }^{2}$ and Julius Ogengo ${ }^{1}$

\begin{abstract}
Background: Africa has $4 \%$ of the global vehicles but accounts for about one tenth of global vehicular deaths. Major trauma in Kenya is associated with excess mortality in comparison with series from trauma centers. The determinants of this mortality have not been completely explored.

Objectives: To determine the factors affecting mortality among road users in Nairobi, Kenya.

Methods: Cross-sectional study of prospectively collected data of trauma admissions at the Kenyatta National Hospital over a calendar year (2009-2010). Information collected included age, gender, road user type, principal anatomical region of injury, admission status, admission blood pressure and GCS, disposition destination, Injury Severity Score (ISS), injuries sustained, treatment and mortality at two weeks. Major or severe injury was defined as injuries of ISS $>15$. Groups based on in-hospital survival were compared using determinants of mortality using $X^{2}$ or students $t$-test as appropriate. Logistic regression was used to assess the independence of predictive variables.
\end{abstract}

Results: One thousand six hundred forty seven (1647) patients were admitted for trauma during the study period. Traffic admissions were 1013 (61.7\%) and males predominated (79.8\%). The average age of patients admitted was 31.7 years. Pedestrians, vehicle occupants and motorcyclists represented $43.3 \%, 27.2 \%$ and $15.2 \%$ of the road users injured. The proportion of patients with ISS $>15$ was 10.9\%.

The overall mortality was $7.7 \%$. Mortality for ISS > 15 was $27.6 \%$. The following factors significantly predicted mortality on univariate analysis: head injury, abdominal injury, transfer in status, blood transfusion, ICU admission, age > 60 years, Glasgow coma scale (GCS) and injury severity. GCS $(p=0.001)$ and ISS $>15(p<0.05)$ remained significant predictors on regression analysis.

Conclusion: Trauma mortality rates in this study exceed those from mature trauma systems. Head injury and injury severity based on the ISS are independent predictors of mortality after traffic trauma. Improvements in neurosurgical and critical care services ingrained within wider primary and secondary prevention initiatives are logical targets.

\section{Introduction}

Road traffic injuries are a global public health problem, with an estimated 1.2 million deaths and 50 million non-fatal injuries per year [1]. Developing countries account for most of these deaths. Highly motorized countries have 60 per cent of global vehicles, but account for only 14 per cent of global deaths. Africa, with only $4 \%$ of the global vehicles, accounts for $11 \%$ of global deaths [2]. In Kenya, 68 deaths per 1,000 registered vehicles are

\footnotetext{
* Correspondence: hsaid2ke@yahoo.com

${ }^{1}$ Department of Human Anatomy, University of Nairobi, Riverside Drive, P.O. Box 30197, Nairobi 00100, Kenya

Full list of author information is available at the end of the article
}

recorded annually (3,000 deaths), a rate 30-40 times that in industrialized countries [3].

A 2000 study showed an unadjusted mortality rate of $35.6 \%$ for severe injuries resulting from road collisions in Kenya's capital, Nairobi [4]. This rate is six times higher than that reported for countries with high income. The determinants of this mortality are incompletely documented. Since the 2000 study, access to advanced trauma life support (ATLS) protocols has improved and policy enhancement towards enforcement of traffic laws [5] embraced. We aimed to study the pattern of road trauma mortality from a hospital perspective and assess the determinants of this mortality using a larger sample of admissions over one calendar year.

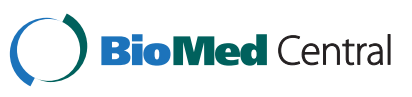




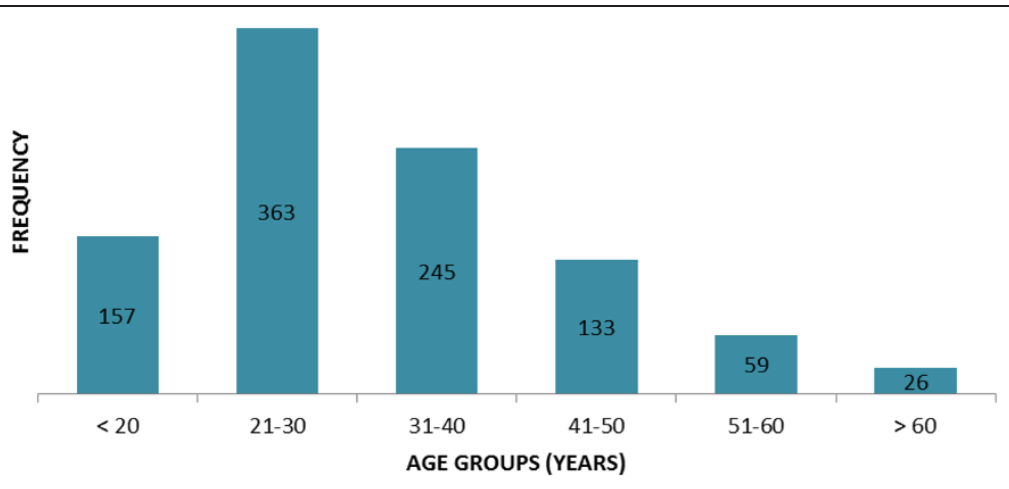

Figure 1 Age distribution for traffic injuries at $\mathrm{KNH}$.

\section{Methods}

Kenyatta National Hospital (KNH) is the largest health facility in Kenya. It is the teaching hospital of the University of Nairobi and has a catchment population of about $3,000,000$ people. The hospital receives most of the trauma in the city and its environs. The study sample included all admissions due to traffic between November 2009 and December 2010. The following factors were recorded: age, gender, road user type, admission from scene or transferin, anatomical region injured, Intensive Care Unit admission, systolic blood pressure on admission, Glasgow Coma Scale (GCS), Injury Severity Score (ISS), treatment and mortality at two weeks. Abbreviated Injury Scale categorization and calculation of Injury Severity Scores were done from the anatomic description of the injuries. Cost data referred to direct cost to the patient as captured from the hospital billing system. Costs are converted to USD from Kenya shillings (conversion rate: 86 shillings to one USD at the time). The mortality information was further evaluated for significant predictors and analyzed (with the Statistical Package for the Social Sciences (version 18,
SPSS Inc. software) using the Chi square test, Fisher's exact test and Student $t$-test as appropriate. A p-value of $<0.05$ was considered significant. Variables analyzed included head/neck involvement, age, gender, injury severity, need for major surgical procedure and transfusion. ISS $>15$ was the threshold for severe injury. Major surgery entailed operations lasting more than one hour and included craniotomies, laparotomies, internal fixation of limb fractures, major wound debridements.

\section{Consent}

Written informed consent was obtained from the patient for the publication of this report and any accompanying images.

\section{Ethical approval}

Ethical approval was obtained from the KNH-UON ethical review committee and the work was done in compliance with the Helsinki Declaration.

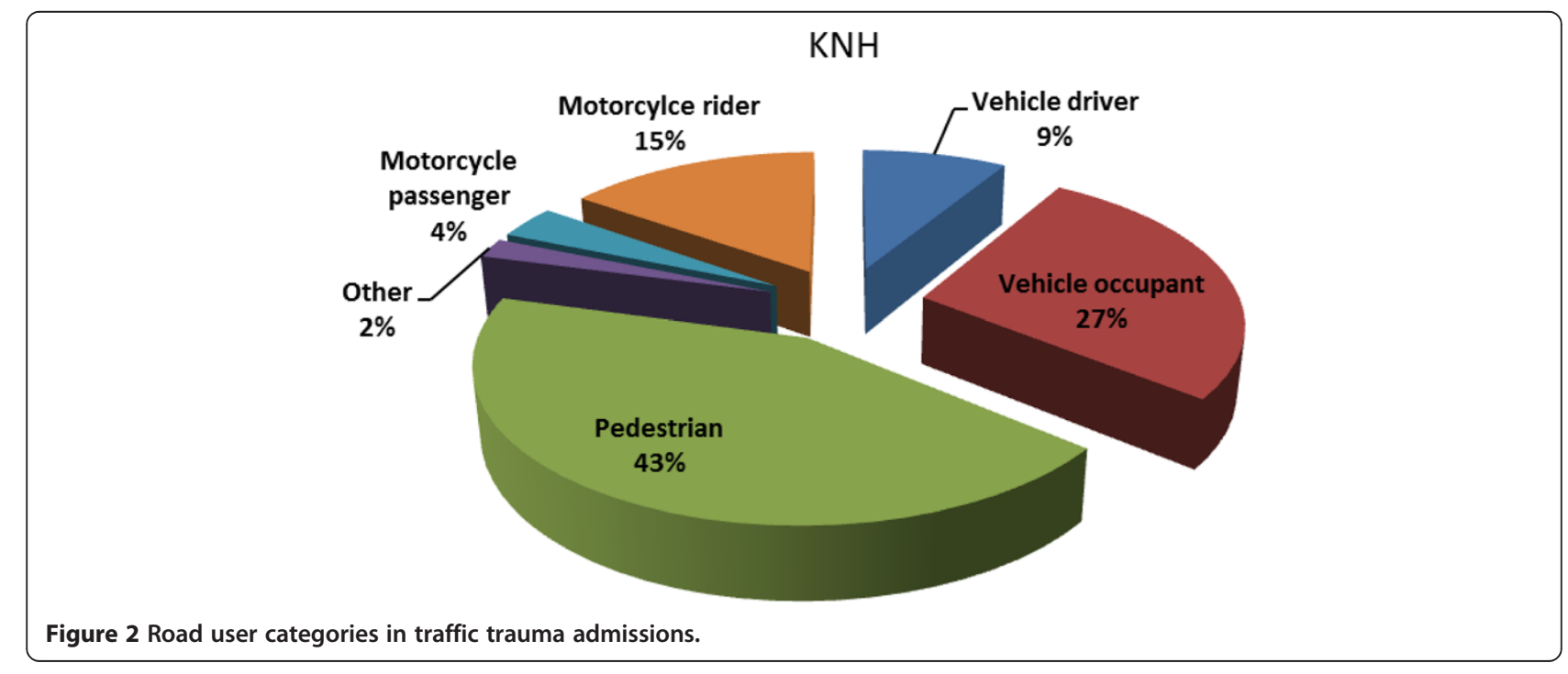


Table 1 Injury severity, length of hospital stay and direct costs by road user type

\begin{tabular}{lllll}
\hline Road user & Mean ISS & Proportion with ISS > 15 & Mean LOS (days) & Mean cost (USD) \\
\hline Motor vehicle driver & $7.2 \pm 3.8$ & $10.5 \%$ & $29.7 \pm 36.8$ & $700.80 \pm 57.51$ \\
Motor vehicle occupant & $7.5 \pm 4.6$ & $15.2 \%$ & $31.4 \pm 43.6$ & $507.87 \pm 73.83$ \\
Pedestrian & $7.0 \pm 4.1$ & $12.6 \%$ & $33.0 \pm 35.3$ & $464.82 \pm 50.31$ \\
Motorcycle passenger & $7.3 \pm 3.6$ & $17.1 \%$ & $33.4 \pm 48.4$ & $487.89 \pm 61.84$ \\
Motorcycle rider & $7.6 \pm 4.0$ & $15.8 \%$ & $21.0 \pm 26.2$ & $301.84 \pm 35.09$ \\
\hline
\end{tabular}

\section{Results}

There were 1647 trauma admissions during the study period and 1013 (61.7\%) were traffic related. Males predominated $(82.4 \%)$. The average age of the patients was 31.7 years (range, 1-98 years, peak age 21-30 years) (Figure 1).

The most common road user injured was the pedestrian (Figure 2) Seat belt use was recorded for 39.3\% of motor vehicle drivers and $11.6 \%$ for occupants. Helmet use was reported for $18.2 \%$ of motorcycle passengers and $45.8 \%$ of riders.

Less than half of the patients (44.5\%) were admitted directly from the crash scene while $55.4 \%$ were transferred from other facilities. The average Injury Severity Score was $7.27 \pm 4.26$ and the proportion with severe injury was $10.9 \%$ overall ((highest for motorcycle passenger) (Table 1)). Six point seven percent were disposed to the ICU from the accident and emergency department.

The most common anatomical regions injured were the extremity (56.4\%) and head/neck (31.2\%). The road users with the highest rate of extremity injuries were motorcycle occupants (62.9\%). Extremity injury rate was $60.5 \%$ for MV driver, $46.23 \%$ for MV occupant, $60.1 \%$ for pedestrian and $61.8 \%$ for motorbike rider. The rate of head and neck injuries were $27.9 \%, 34.1 \%, 28.8 \%$, $25.7 \%$ and $34.9 \%$ for motor vehicle drivers, motor vehicle occupants, pedestrians, motor cycle occupants and motor cycle riders respectively (Table 2). Of 315 injuries in the head/neck, $200(63.5 \%)$ were isolated to the head, $28(8.9 \%)$ isolated to the face and the rest were multiple injuries.

The mean hospital stay was $30.4 \pm 37.5$ days (range 1-130, highest for motorcycle passenger).
Overall mortality was 7.7\%. Mortality was $9.5 \%$ for motorcycle rider, $8.6 \%$ for MVC and motorcycle occupant, $7.5 \%$ for pedestrian and 3.6\% for MVC driver.

Factors that were significantly associated with hospital mortality on univariate analysis were head involvement, ICU admission, non-surgical treatment and blood transfusion $(\mathrm{p}<0.01)$. Additionally, mortality was significantly influenced by transfer status at admission $(\mathrm{p}=0.01)$, abdominal injury $(\mathrm{p}=0.011)$, injury severity score $(\mathrm{p}<0.001)$ and GCS $(p<0.001)$. Age $(p=0.07)$, gender $(p=0.38)$ and admission BP $(\mathrm{p}=0.96)$ and individual road user category did not influence mortality (Tables 3 and 4).

\section{Discussion}

This study has shown that road injuries continue to be the biggest component of the trauma burden at $\mathrm{KNH}$ and are associated with significant rate of death. In this study $7.7 \%$ of patients ( $43.6 \%$ of these pedestrians, $34.2 \%$ occupants, $22.0 \%$ of motorcycle users) died within fourteen days of admission. Although no age group was immune, the peak age of individuals in their third decade of life underscore the significant economic burden of road crashes [4]. The cost of the injury due to traffic injuries, computed from treatment costs and lost productivity, has been estimated to be equivalent to $1-2 \%$ of GDP of most countries [6].

The other results on trauma demography echo similar statistics in the trauma literature from low income countries where males, pedestrians and motorcyclists are most vulnerable [7]. The characteristics of the male thought to contribute significantly to the gender bias include a risk taking behavior and younger age. The predominant involvement of pedestrians and motorcyclists in

Table 2 Predominant region of injury versus road use category at KNH

\begin{tabular}{|c|c|c|c|c|c|c|c|}
\hline Injury & MVC driver (\%) & MVC occupant (\%) & Pedestrian (\%) & Other (\%) & MC occupant (\%) & MC rider (\%) & Total \\
\hline Head/neck & $24(7.7)$ & $83(29.7)$ & $124(38.6)$ & $10(3.25)$ & $9(16.9)$ & $53(16.9)$ & 313 \\
\hline Chest & $1(3.1)$ & $14(43.8)$ & $15(46.9)$ & 0 & $1(3.1)$ & $1(3.1)$ & 32 \\
\hline Abdomen & $2(6.1)$ & $7(21.2)$ & $22(66.7)$ & 0 & 0 & $2(6.1)$ & 33 \\
\hline Spine & $7(14.6)$ & $31(64.6)$ & $5(10.4)$ & $1(2.1)$ & $2(4.2)$ & $2(4.2)$ & 48 \\
\hline External & 0 & $1(12.5)$ & $6(75.0)$ & 0 & $1(12.5)$ & 0 & 8 \\
\hline Extremity & $52(9.2)$ & $126(22.4)$ & $259(46.0)$ & $10(1.8)$ & $22(3.9)$ & $94(16.7)$ & 563 \\
\hline Total & $86(8.6)$ & $272(27.4)$ & $431(43.2)$ & $21(2.1)$ & $35(3.5)$ & $152(15.2)$ & 997 \\
\hline
\end{tabular}


Table 3 Univariate analysis of factors influencing mortality after traffic trauma

\begin{tabular}{|c|c|c|c|c|c|}
\hline Variable & & Alive & Died & $P$ value & OR $(\mathrm{C} / \mathrm{I})$ \\
\hline \multirow[t]{2}{*}{ Disposition } & Wards & 841 & $41(4.6 \%)$ & $<0.001$ & $10.1(6.0-16.8)$ \\
\hline & ICU/OR & 71 & $35(33.0 \%)$ & & \\
\hline \multirow[t]{2}{*}{ Region of injury } & Head/neck & $263(85.4 \%)$ & $45(14.6 \%)$ & $<0.001$ & $3.2(2.1-4.9)$ \\
\hline & Other & 649 (95.4\%) & $31(4.6 \%)$ & & \\
\hline \multirow[t]{2}{*}{ Surgical treatment } & Major procedure & $452(96.8 \%)$ & $15(3.2 \%)$ & $<0.001$ & $3.9(2.2-7.0)$ \\
\hline & Nonsurgical care & 446 (88.5\%) & $11.5 \%$ & & \\
\hline \multirow[t]{2}{*}{ Injury severity } & $I S S \leq 15$ & 806 (95.4\%) & $39(4.6 \%)$ & $<0.001$ & $7.9(4.8-12.9)-$ \\
\hline & ISS $>15$ & $97(72.4 \%)$ & $37(27.6 \%)$ & & \\
\hline \multirow[t]{2}{*}{ Admission status } & Direct from scene & $383(95.1 \%)$ & $20(4.9 \%)$ & 0.001 & $2.5(1.5-4.3)$ \\
\hline & Transfer-in & 409 (88.5\%) & $53(11.5 \%)$ & & \\
\hline \multirow[t]{2}{*}{ Age } & $<60$ years & 862 (92.9\%) & $66(7.1 \%)$ & 0.07 & $2.4(0.9-6.5)$ \\
\hline & $\geq 60$ years & $27(84.4 \%)$ & $5(15.6 \%)$ & & \\
\hline \multirow[t]{2}{*}{ Gender } & Male & 718 (92.1\%) & $62(7.9 \%)$ & 0.38 & $1.3(0.7-2.4)$ \\
\hline & Female & 185 (93.9\%) & $12(6.1 \%)$ & & \\
\hline \multirow[t]{2}{*}{ Blood product } & Transfused & 142 (82.6\%) & $30(17.4 \%)$ & $<0.001$ & $2.6(1.7-4.1)$ \\
\hline & Not transfused & 641 (93.4\%) & $45(6.6 \%)$ & & \\
\hline \multirow[t]{4}{*}{ Specific injury } & Head injuries & $173(84.6 \%)$ & $31(15.2 \%)$ & $<0.001$ & $2.6(1.7-4.0)$ \\
\hline & No head injury & 732 (94.2\%) & $45(5.8 \%)$ & & \\
\hline & Abdominal injury & $18(78.3 \%)$ & $5(21.7 \%)$ & 0.011 & $2.9(1.3-6.6)$ \\
\hline & No abdominal injury & 887 (92.6\%) & $71(7.4 \%)$ & & \\
\hline
\end{tabular}

this study differs from road user patterns in high income countries where vehicle occupants predominate. In the United Arab Emirates, a high income developing country, drivers are the most frequently injured road users. Middle to high income Emirati formed a big proportion of vehicle occupants while $88 \%$ of injured pedestrian were non-Emirati from low income countries [8]. Injury prevention initiatives locally should therefore prioritize pedestrian and motorcyclist safety through educational, engineering and legislative programs, proven to reduce road trauma events $[7,9]$. These may include enforcement of speed laws, motorcycle and pedestrian lanes, use of seat belts, helmets and general public education on traffic safety. Intervention targeting motorcyclists have shown that the use of helmets reduce trauma mortality [9] while protective attire including padded suits and boots may

Table 4 Influence of mean ISS, BP, GCS and age on mortality for traffic injury

\begin{tabular}{llll}
\hline & Alive & Died & $\begin{array}{l}\text { P value }(C / I \text { of } \\
\text { mean difference })\end{array}$ \\
\hline Age (years) & 31.68 & 33.85 & $0.21(-5.5-1.2)$ \\
Admission GCS & 14.01 & 10.35 & $<0.001(2.4-4.9)$ \\
Admission BP & 117.01 & 117.11 & $0.96(-4.5-4.3)$ \\
ISS & 6.88 & 11.58 & $<0.001(-5.6--3.7)$ \\
\hline
\end{tabular}

impact on the morbidity to the limbs that follow motorcycle crashes. Kenya recently experienced the evidence that the road carnage can indeed be controlled using local mechanisms modified from their proven track records in high income countries. When introduced for a short period in 2004, 'Michuki laws' that enforced traffic rules in the public transportation sector was associated with a reduction in hospital admission and numbers of fatal and serious injuries [5].

The average hospital stay was long (30.4 days) for our patients. Comparatively, hospital stay was significantly shorter (9.7 days) for patients in the UAE even with higher acuity trauma (a greater proportion needing ICU care (13\% versus $6 \%$ in the current study) for UAE patients [8]. The explanation for the KNH statistics lies in the policy of fixation of skeletal fractures adopted. Most patients sustaining limb fractures cannot afford implants and the timing of fixation influenced by when families can raise enough funds to access implants. Rigorous cost-effectiveness assessment of this local resource dynamic must be undertaken so that an informed position on policy change to early fixation can be embraced. This policy reorientation must be guided by the potential economic productivity of the young patients who could go back to work earlier and that savings may be made by policy of early fracture fixation by reducing complications associated with prolonged hospitalization. 
The overall and major trauma mortality was $7.7 \%$ and $27 \%$ respectively. This compares unfavorably with trauma mortality rate in high income countries. Although major trauma mortality has improved from a decade ago (36\%), the current rate is still five times higher from rate in high income countries [10]. Differences in resources and advances in organization of trauma care may explain the disparate rates $[11,12]$. There is a relationship between a country's level of income and mortality; higher incomes are associated with declining mortality $[8,13]$. This presumably must relate to levels of investments in the trauma care infrastructure. The specific investments have included maturation of trauma systems, enhanced protocols in trauma resuscitation including damage control surgery, lung protective ventilation and traumatic brain injury. Between 1996-2008 for example, mortality in one trauma center in the USA ranged from 3 to $3.7 \%$ even as the center handled a higher proportion of older patients and worse injuries than in our study [14]. Nairobi lacks an organized trauma system at the present moment. Serious efforts are needed to put in place a trauma care system that can optimize outcomes.

This study has shown that head involvement, GCS (GCS was 10.0 for non-survivors but 14.0 for survivors), nonsurgical treatment, blood transfusion, transfer-in status and injury severity were significant predictors of early mortality at univariate analysis but only low GCS and injury severity were independent predictors on logistic regression. The result on head injury and GCS is consistent with previous studies [4]. We contend that the other variables lost in the logistic regression are useful proxies to injury severity and head involvement. Admission to the ICU, blood transfusion, transfer-in and non-surgical status are associated with severe illness even in settings outside trauma. Patients with head injury are the more likely trauma victims to be transferred to KNH for specialized investigations and critical. There is need to study whether this is the case and strategies to improve head trauma services.

The result on blood pressure was surprising. Several other series have shown that this modifiable variable is a predictor of trauma mortality $[8,15]$. Possible explanations include the fact the study population was young (threshold for physiological derangements higher) and that this initial vital sign was not recorded for some patients. Although hypotension is reversible and could explain the result, we contend that sustained hypotension is a marker of injury severity and trauma protocols should still take the vital sign seriously.

The interactions of age, gender and road user type with mortality were not significant. This is also consistent with other studies. The implication of this is that all trauma should continue to be treated along similar lines initially (primary survey) for both gender and mechanisms.
This study had limitations. As stated earlier, the manner in which skeletal injury is managed at KNH limits comparisons with trauma centers. Secondly, this was a non-registry data analysis and is tedious. To enable the institution continuously interrogate its trauma performance, a registry system should be developed to enhance continuous evaluation of care. We have not analyzed the risk factors for road trauma including alcohol, speeding and restraint, known for their impact on road trauma mortality [16] and important ingredients for the development of prevention measures.

In conclusion, the trauma mortality rate at $\mathrm{KNH}$ is comparatively high. The independent predictors of mortality are GCS and injury severity score. The effects of neurotrauma and complex injury can be mitigated by developing a responsive trauma system including a registry system and critical care.

\section{Competing interests}

The authors declare that they have no competing interests.

\section{Authors' contributions}

HS conceptualized the work and participated in the overall study organization, data collection, analysis, manuscript writing. JO participated in the study conceptualization and drafting of the manuscript. BKM was involved in the study organization, data collection, analysis, drafting of the manuscript. All the authors read and approved the final manuscript.

\section{Author details}

'Department of Human Anatomy, University of Nairobi, Riverside Drive, P.O. Box 30197, Nairobi 00100, Kenya. ${ }^{2} E m b u$ Provincial General Hospital, P.O. Box 33, Embu 60100, Kenya.

Received: 13 December 2012 Accepted: 4 February 2014

Published: 13 February 2014

\section{References}

1. World Health Organization: Global status report on road safety: time for action. 2009. (http://www.who.int/violence_injury_prevention/road_safety_status/2009/en/).

2. Jacobs GD, Aeron-Thomas A: A review of global road accident fatalities. 2012. http://www.transport-links.org/transport_links/filearea/publications/1_771_pa3568. pdf accessed February 21, 2014.

3. Odero W, Khayesi M, Heda P: Road traffc injuries in Kenya: magnitude, causes and status of intervention. Injury and Safety Promotion J 2003, 10(1-2):53-62.

4. Saidi H, Macharia WM, Atinga JEO: Outcome for hospitalized road trauma patients at a tertiary hospital in Kenya. Eur J Trauma, 31(4):401-406.

5. Chitere PO, Kibua TN: Efforts to improve road safety in Kenya. Achievements and limitations of reforms in the Matatu industry; 2012. http://www.ssatp. org/sites/ssatp/files/publications/CountryDocuments/Road-Safety-KenyaIPAR.pdf accessed February 21, 2014.

6. Peden M, Scarfield R, Sleet D, Mohan D, Hyder A, Jarawan E, et al: World report on road traffic injury prevention. Geneve: World Health Organization; 2004

7. Mock C, Quansah R, Krishnan R, Arreola-Risa C, Rivara F: Strengthening the prevention and care of injuries worldwide. Lancet 2004, 363:2172-2179.

8. Eid HO, Barss P, Adam SH, Torab FC, Lunsjo K, Grivna M, Abu-Zidan FM: Factors affecting anatomical region of injury, severity and mortality for road trauma $\mathrm{n}$ a high income developing country: lessons for prevention. Injury 2009, 40(7):703-707.

9. Mock C, Kobusingye O, Anh LV, Afukar F, Arreola-Risa C: Human resources for the control of road traffic injury. Bull World Health Org. 2005, 83:294-300

10. Mock CN, Jurkovich GJ, Nii-Amon-Kotei D, Arreola-Risa C, Maier RV: Trauma mortality patterns in three nations at different economic levels: implications for global trauma system development. J Trauma 1998, 44(5):804-812. 
11. Mann N, Mulins R, Mackenzie E, Jurkovich G, Mock V: A systematic review of published evidence regarding trauma system effectiveness. J Trauma 1999, 47:525-533.

12. MacGowan WA: Surgical manpower worldwide. Bull Amer Coll Surg 1987, 72:5-9.

13. Mock CN, Adzotar KE, Conklin E, Denno DM, Jurkovich GJ: Trauma outcomes in the rural developing world: comparison with an urban level trauma center. J Trauma 1993, 35:518-523.

14. Dutton RP, Stansbury LG, Leone SBA, Kramer ERN, Hess JR, Scalea TM: Trauma mortality in mature trauma systems: are we doing better? an analysis of trauma mortality patterns, 1997-2008. J of Trauma-Injury Infec \& Critical Care 2010, 69(3):620-626.

15. Hasler RM, Nuesch E, Jüni P, Bouamra O, Exadaktylos AK, Lecky F: Systolic blood pressure below $110 \mathrm{~mm} \mathrm{Hg}$ is associated with increased mortality in blunt major trauma patients: multicentre cohort study. Resuscitation 2011, 82(9):7-1202

16. Qirjako G, Burazeri G, Hysa B, Roshi E: Factors associated with fatal traffic accidents in Tirana, Albania: cross-sectional study. Croat Med J 2008, 49(6):734-740.

doi:10.1186/1752-2897-8-4

Cite this article as: Saidi et al:: Mortality after road traffic crashes in a system with limited trauma data capability. Journal of Trauma

Management \& Outcomes 2014 8:4.

\section{Submit your next manuscript to BioMed Central and take full advantage of:}

- Convenient online submission

- Thorough peer review

- No space constraints or color figure charges

- Immediate publication on acceptance

- Inclusion in PubMed, CAS, Scopus and Google Scholar

- Research which is freely available for redistribution 